\title{
KRULL AND GLOBAL DIMENSIONS OF FULLY BOUNDED NOETHERIAN RINGS
}

\author{
KENNETH A. BROWN AND R. B. WARFIELD, JR.
}

\begin{abstract}
The main result of this paper states that the Krull dimension of a fully bounded Noetherian ring containing an uncountable central subfield is bounded above by its global dimension, provided that the latter is finite.
\end{abstract}

The proof requires some results on projective dimensions and on localization (Corollary 4 and Theorem 11, respectively), which may be of independent interest. If $P$ is a prime ideal in a Noetherian ring $R$, then $P$ is contained in a unique clique, $X$, a subset of $\operatorname{Spec}(R)$ defined below (Definition 6). Now in some circumstances, the set $C(X)$ of elements of $R$ regular modulo every element of $X$ is an Ore set in $R$, and the localized ring $R_{X}$ obtained by inverting the elements of $C(X)$ has certain desirable properties. In this case, $X$ is said to be classical (Definition 7). We prove in Theorem 8 that if $R$ is a Noetherian ring of finite global dimension whose cliques are classical, then the classical Krull dimension of $R$ is bounded above by its global dimension. Generalizing work of B. J. Mueller and A. V. Jategaonkar $[\mathbf{1 6}, \mathbf{1 3}]$, we show that if $R$ is a Noetherian fully bounded ring containing an uncountable set $F$ of central units such that the difference of two distinct elements of $F$ is still a unit, then all cliques in $\operatorname{Spec}(R)$ are classical (Theorem 11). This applies, in particular, if $R$ has an uncountable central subfield, as in the abstract. Using Theorems 8 and 11 in their general forms, K. R. Goodearl and L. W. Small have shown that the inequality of Krull and global dimensions is true for all Noetherian P. I. rings [10].

In this paper, all modules are right modules unless it is indicated otherwise. If $M$ is a module, then $r(M)$ is the right annihilator of $M$ and (if appropriate) $l(M)$ is the left annihilator of $M$. A ring is Noetherian if it satisfies the ascending chain condition on right and left ideals. If $P$ is a prime ideal of $R$, then $R / P$ is right bounded if every essential right ideal of $R / P$ contains a nonzero two-sided ideal. A ring $R$ is fully bounded Noetherian (abbreviated FBN) if it is Noetherian and for every prime ideal $P, R / P$ is both right and left bounded.

Part of this research was done while the first author held a visiting position at the University of Washington. He is grateful to that institution for its hospitality. The research of the second author was supported in part by a grant from the NSF.

\section{Homological results.}

LEMMA 1. Let $R$ be a ring, $A$ a right $R$-module, and $M$ a maximal co-Artinian ideal of $R$. Then for every nonnegative integer $n, \operatorname{Ext}_{R}^{n}(A, R / M)=0$ if and only if $\operatorname{Tor}_{R}^{n}(A, R / M)=0$.

Received by the editors December 2, 1983.

1980 Mathematics Subject Classification. Primary 16A33; Secondary 16A60.

Key words and phrases. Noetherian rings, global dimension, Krull dimension, fully bounded rings, localization, cliques. 
ProOF. Note that $R / M$ is simple Artinian and is thus an injective cogenerator in the category of $R / M$-modules. The statement thus follows from [5, Proposition 5.1, p. 120]. A direct proof can easily be given using a projective resolution of $A$ and the fact that for any $R$-module $B, B \otimes(R / M)$ is naturally isomorphic to $B / B M$.

Proposition 2. Let $R$ be a Noetherian ring and $A$ a finitely generated $R$ module of finite projective dimension. Suppose that every primitive ideal of $R$ is co-Artinian. Then pr.dim. $A=n$ if and only if (i) $\operatorname{Ext}_{R}^{n+i}(A, X)=0$ for all irreducible right $R$-modules $X$ and all $i \geq 1$, and (ii) there exists an irreducible right module $Y$ such that $\operatorname{Ext}_{R}^{n}(A, Y) \neq 0$.

PROOF. This is immediate from Lemma 1 and [3, Theorem 2.1].

It would be interesting to know which rings satisfy the conclusion of Proposition 2. Not all hereditary Noetherian domains do so-consider a non-Artinian one with all its irreducible modules injective [6].

LEMMA 3. Let $R$ be a Noetherian ring of finite global dimension $k$ such that, for every co-Artinian ideal $I$ of $R$, every finitely generated submodule of the right injective hull of $R / I$ has a co-Artinian annihilator. Let $P$ be a nonmaximal prime ideal of $R$. Then $\operatorname{Ext}^{k}(R / P, R / I)=0$ for all co-Artinian ideals $I$ of $R$.

ProOF. Suppose there exists a co-Artinian ideal $I$ with $\operatorname{Ext}^{k}(R / P, R / I) \neq$ 0 . Let $E_{k}$ be the $k$ th term of a minimal injective resolution of $(R / I)_{R}$, and let $E=\operatorname{Ext}^{k}(R / P, R / I)$. By hypothesis, every finitely generated submodule of $E_{k}$ has co-Artinian annihilator. Now $E$ is a subfactor of $\operatorname{Hom}_{R}\left(R / P, E_{k}\right.$ ) (as a right $R$-module). If $g \in E$, then $g$ is the image of an element $f, f \in \operatorname{Hom}_{R}\left(R / P, E_{k}\right)$, and there is a co-Artinian ideal $A$ with $P \subset A$ and $f(R / P) A=0$. Hence

$$
f A(R / P)=f(A+P / P)=f(R / P) A=0,
$$

so $f A=0$, and, therefore, $g A=0$ in $E_{R}$. Since $R$ is right Noetherian, ${ }_{R} E$ is finitely generated, so there is a co-Artinian ideal $A$ with $E A=0$. Since $P$ is not maximal, there exists an element $\alpha$ with $\alpha \in A \cap C(P)$. The exact sequence

$$
0 \rightarrow R / P \stackrel{\alpha}{\rightarrow} R / P \rightarrow R / P+x R \rightarrow 0
$$

yields an exact sequence

$$
E \stackrel{\alpha^{*}}{\rightarrow} E \rightarrow \operatorname{Ext}^{k+1}(R / P+x R, R / I)
$$

and the right-hand term in (3) is zero by hypothesis. Now $\alpha^{*}$ is right multiplication by $\alpha$ on $E$ (to see this, take an injective resolution of $R / I$ ). Since $\alpha \in A, E a=0$, while (3) shows that $E \alpha=\alpha^{*}(E)=E$. Hence $E=0$.

COROLlaRY 4. Let $R$ be a Noetherian ring of finite global dimension $k$, and such that (i) every primitive ideal is co-Artinian, and (ii) every finitely generated submodule of the right injective hull of a simple right $R$-module has co-Artinian annihilator. Let $P$ be a nonmaximal prime ideal of $R$. Then pr. $\operatorname{dim} .(R / P)_{R}<k$.

COROLlaRY 5. Let $R$ be a fully bounded Noetherian ring of finite global dimension $k$. Let $X$ be a finitely generated $R$-module with no Artinian submodules. Then pr.dim. $(X) \leq k-1$.

ProOF. There is a finite chain of submodules of $X$ with each factor (Krull) critical, not Artinian, and having prime annihilator $[\mathbf{1 2}, 2.5$ and 3.2]. It is clearly 
enough to prove the result when $X$ is itself such a critical module, with prime annihilator $P$. By $[\mathbf{1 2}, 1.1(\mathrm{~b})$ and 2.5] there is an exact sequence of right $R$-modules

$$
0 \rightarrow X \rightarrow R / P \rightarrow W \rightarrow 0 \text {. }
$$

Let $M$ be a maximal ideal of $R$. From (4) we get an exact sequence

$$
\operatorname{Ext}^{k}(R / P, R / M) \rightarrow \operatorname{Ext}^{k}(X, R / M) \rightarrow \operatorname{Ext}^{k+1}(W, R / M)
$$

The outer terms of (5) are zero by Lemma 3 and by hypothesis. Thus $\operatorname{Ext}^{k}(X, R / M)$ $=0$ and so pr. $\operatorname{dim} .(X) \leq k-1$ by Proposition 2 .

NOTES. (i) Corollary 5 generalizes a well-known and easy result for commutative Noetherian rings. Even in this case, the hypothesis that $X$ is finitely generated is clearly needed-consider $R=Z, X=Q$.

(ii) There is no generalization of Corollary 5 to rings satisfying one-sided hypotheses only; consider, for example, the rings constructed in [8 and 11].

2. Cliques and localization. If $R$ is a Noetherian ring and $P$ and $Q$ are prime ideals of $R$, we say there is a second layer link $Q \rightsquigarrow P$ if there is an ideal $J$, with $Q \cap P \supset J \supseteq Q P$, such that $J \neq Q \cap P$ and $Q \cap P / J$ is torsion-free as a left $R / Q$-module and as a right $R / P$-module. If $R$ is an FBN ring, then such a $J$ will exist precisely when $Q=l(Q \cap P / Q P)$ and $P=r(Q \cap P / Q P)$, and in this case $R / P$ and $R / Q$ must have the same Krull dimension. (These links were introduced by Jategaonkar and are studied in [15 and 13, Chapter 5].)

DEFINITION 6. The graph of links of $R$ is the directed graph whose vertices are the elements of $\operatorname{Spec}(R)$ and whose arrows are given by the second layer links. A clique in $\operatorname{Spec}(R)$ is a connected component of the graph of links.

Definition 7. If $X$ is a clique in $\operatorname{Spec}(R)$ and $C(X)=\bigcap\{C(Q): Q \in X\}$, then $X$ is classical if (i) $C(X)$ is a right and left Ore set, so that a localization $R_{X}=R C(X)^{-1}$ exists, (ii) for every prime $Q, Q \in X, R_{X} / Q R_{X}$ is naturally isomorphic to the Goldie quotient ring of $R / Q$, (iii) the primes $Q R_{X}, Q \in X$, are precisely the primitive ideals of $R_{X}$, and (iv) the $R_{X}$ injective hull of every simple $R_{X}$-module is the union of its socle sequence.

This definition is taken from [13, Chapter 8], in which there is an extended discussion of cliques and localization.

THEOREM 8. Let $R$ be a Noetherian ring of finite global dimension whose cliques are classical. Then $\mathrm{cl} . \mathrm{K} . \operatorname{dim} R \leq \mathrm{gl} . \operatorname{dim} . R$.

Proof. Choose a chain of distinct prime ideals $P_{0} \supset P_{1} \supset \cdots \supset P_{k}$. Let $X$ be the clique containing $P_{0}$. Note that gl. dim. $R_{X} \leq$ gl. $\operatorname{dim} . R$ and $P_{0} R_{X} \supset P_{1} R_{X} \supset$ .. $\supset P_{k} R_{X}$ is again a chain of distinct primes of $R_{X}$. We will show that $k \leq$ gl. $\operatorname{dim} . R_{X}$. In effect, this means that we may assume in advance that $P_{0}$ is a coArtinian ideal. Assuming this, we now let $Y$ be the clique containing $P_{1}$. If $Q \in Y$, then $R / Q$ is not Artinian, since $R / P_{1}$ is not Artinian and this property is preserved by bimodule links [14]. Hence by Corollary 4 , for $Q \in Y$, pr. dim. $R / Q<\operatorname{gl}$. dim. $R$. Now $R_{Y}$ has finite global dimension and its simple modules are all summands of localizations of the modules $R / Q, Q \in Y$, so we conclude (using $[3,2.1$ or 1 or $\mathbf{9}$, Theorem 16]) that gl. dim. $R_{Y}<$ gl. dim. $R$. By induction, since the ideals $P_{i} R_{Y}$ are all prime ideals in $R_{Y}(i>0)$, we conclude that $k \leq$ gl. dim. $R$, as required. 
At present, no FBN ring is known to have a clique which is not classical. Finite cliques in FBN rings are classical [15, Theorem 5 or 13, 8.8]. A Noetherian ring whose cliques are all finite and classical is said to have enough clans. For such rings (not necessarily FBN). Theorem 8 has been obtained as [3, Theorem 4.9]. The cliques of a Noetherian P.I. ring $R$ containing an uncountable field are shown to be classical in $[\mathbf{1 2}, 8.45]$ (or [16] for the case where $R$ is affine over $F$ ). Theorem 11 below gives an easier proof of this, at the same time extending it to a wider class of rings.

DEFINITION 9. A nonempty set $X$ of prime ideals in a ring $R$ satisfies the intersection condition if every one-sided ideal of $R$ which has nonempty intersection with $C(P)$ for all $P \in X$ also has nonempty intersection with $C(X)$.

LEMMA 10. Let $R$ be a fully bounded Noetherian ring and let $X$ be a countable subset of $\operatorname{Spec}(R)$. Assume that $R$ contains an uncountable set $F$ of central units such that the difference of any two distinct elements of $F$ is a unit. Then $X$ satisfies the intersection condition.

Proof. We first remark that if we partition $X$, say $X=X_{1} \cup X_{2}$, and if for some right ideal $I$ we can show that $I \cap C\left(X_{1}\right) \neq \emptyset$ and $I \cap C\left(X_{2}\right) \neq \emptyset$, then it follows that $I \cap C(X) \neq \emptyset$. For suppose that $w_{1} \in I \cap C\left(X_{1}\right)$ and $w_{2} \in I \cap C\left(X_{2}\right)$. Note that the natural map $F \rightarrow R / P$ is one-to-one, for every prime ideal $P$. Hence, we infer from [13, Lemma 8.42] that for a particular prime $P, P \in X_{1} \cup X_{2}$, there are at most finitely many $\alpha \in F$ for which $\alpha w_{1}+w_{2}$ is not regular modulo $P$. Since $X_{1} \cup X_{2}$ is countable while $F$ is uncountable, there is an $\alpha \in F$ such that $\alpha w_{1}+w_{2}$ is regular modulo every prime ideal in $X_{1} \cup X_{2}$.

Applying this argument a finite number of times, we reduce to the case in which $R$ is a prime ring and $\bigcap\{P: P \in X\}=0$. Suppose that $I$ is a right ideal of $R$ such that $I \cap C(P) \neq \emptyset$ for all $P \in X$. Then in particular, the left and right annihilators of $I$ are contained in all the ideals in $X$, and thus are zero. Therefore $I$ is an essential right ideal of $R$, and contains a regular element $x$. Since $R$ is right fully bounded, there is a nonzero ideal $J$ contained in $x R$. Let $X_{1}=\{P \in X: P \nsupseteq J\}$ and $X_{2}=\{P \in X: P \supseteq J\}$. If $P \in X_{1}$, then $P+J / P$ is a nonzero ideal of $R / P$, contained in the right ideal generated by $x+P$, and therefore $I \cap C\left(X_{1}\right) \neq \emptyset$. (The authors are indebted to $\mathrm{K} .^{\prime} \mathrm{R}$. Goodearl for this observation, which considerably simplifies their previous proof of this result.) Since the ideals in $X_{2}$ all contain $J$, it follows by Noetherian induction that $I \cap C\left(X_{2}\right) \neq \emptyset$. By the argument in the first paragraph, we again conclude that $I \cap C(X) \neq \emptyset$. This completes the proof of Lemma 10.

THEOREM 11. Let $R$ be a fully bounded Noetherian ring which contains an uncountable set $F$ of central units such that the difference of any two distinct elements of $F$ is a unit. Then all cliques in $\operatorname{Spec}(R)$ are classical.

PROOF. A result of Stafford's [18 or 13, 7.18] shows that the second layer link graph of $R$ is locally finite. It follows immediately that every clique in $\operatorname{Spec}(R)$ is countable, and Lemma 10 shows that every clique satisfies the intersection condition. If $X$ is a clique in $\operatorname{Spec}(R)$ and $P$ and $Q$ are in $X$, then the well-known results of [12] imply that $R / P$ and $R / Q$ have the same Krull dimension, so $P$ and $Q$ must be incomparable. Now an important result of Jategaonkar's [13, 8.35 or 2] shows that a clique $X$ in a Noetherian ring is classical if and only if (i) it satisfies 
the intersection condition, (ii) the elements of $X$ are incomparable, and (iii) the elements of $X$ satisfy a technical condition, the second layer condition. We have already shown that (i) and (ii) hold in our situation. We will not discuss the second layer condition, except to remark that as defined in [13 or 2], it rests upon whether certain modules are wild, and for an FBN ring $R$, there are no wild modules. Thus (iii) is satisfied for all FBN rings. This completes our proof of Theorem 11.

COROLLARY 12. If $R$ is a fully bounded Noetherian ring containing an uncountable set $F$ of central units, such that the difference of any two distinct elements of $F$ is a unit, then

$$
\mathrm{K} . \operatorname{dim} .(R) \leq \operatorname{gl} . \operatorname{dim} .(R) .
$$

This applies in particular to a Noetherian P.I. ring which is an algebra over an uncountable field. Goodearl and Small [10] have used this result to prove that for any Noetherian P.I. ring $R, \mathrm{~K} . \operatorname{dim} .(R) \leq \operatorname{gl} . \operatorname{dim} .(R)$.

In general, the above inequality is strict, even when $R$ is prime and affine $[\mathbf{1 7}$, Example 1.8]. It seems possible, however, that equality may hold for an FBN ring $R$ of finite global dimension such that any two irreducible $R$-modules whose annihilators are in the same clique have the same projective dimension. This condition is certainly sufficient when $R$ is a finite module over its center $[\mathbf{4}, 2.5]$. But even when $R$ is an FBN ring of finite global dimension whose cliques are all singletons-that is, $R$ is an $A R$-ring - we have been unable to prove the equality of the Krull and global dimensions. Precisely this last case is stated as Theorem 7 of $[\mathbf{7}]$, but there appears to be a gap in the proof of this result at p. 58, lines 7-8.

\section{REFERENCES}

1. S. M. Bhatwadekar, On the global dimension of some filtered algebras, J. London Math. Soc. (2) 13 (1976), 239-248.

2. K. A. Brown, Ore sets in Noetherian rings, Seminarie Malliavin (to appear).

3. K. A. Brown, C. R. Hajarnavis and A. E. MacEacharn, Noetherian rings of finite global dimension, Proc. London Math. Soc. (3) 44 (1982), 349-371.

4. K. A. Brown and C. R. Hajarnavis, Homologically homogeneous rings, Trans. Amer. Math. Soc. 281 (1984), 197-208.

5. H. Cartan and S. Eilenberg, Homological algebra, Princeton Univ. Press, Princeton, N.J., 1956.

6. J. H. Cozzens, Homological properties of the ring of differential polymomials, Bull. Amer. Math. Soc. 76 (1970), 75-79.

7. R. F. Damiano and Z. Papp, Stable rings with finite global dimension, Advances in Non-Commutative Ring Theory (Proc. Plattsburgh, 1981), Lecture Notes in Math., vol. 951, SpringerVerlag, Berlin, 1982, pp. 47-67.

8. K. L. Fields, On the global dimension of residue rings, Pacific J. Math. 32 (1970), 345-349.

9. K. R. Goodearl, Global dimension of differential operator rings. II, Trans. Amer. Math. Soc. 209 (1975), 65-85.

10. K. R. Goodearl and L. W. Small, Krull versus global dimension in noetherian P.I. rings, Proc. Amer. Math. Soc. 92 (1984), 175-178.

11. A. V. Jategaonkar, A counterexample in ring theory and homological algebra, J. Algebra 12 (1969), 418-440.

12. __ Jacobson's conjecture and modules over fully bounded Noetherian rings, J. Algebra 30 (1974), 103-121.

13. L L L Lalisation in Noetherian rings (to appear)

14. T. H. Lenagan, Artinian ideals in Noetherian rings, Proc. Amer. Math. Soc. 51 (1975), 499-500.

15. B. J. Mueller, Localisation in fully bounded Noetherian rings, Pacific J. Math. 67 (1976), 233-245. 
16. _ Two-sided localisation in Noetherian PI-rings, Ring Theory, Lecture Notes in Pure and Appl. Math., vol. 51, Marcel Dekker, New York, 1979, pp. 169-190.

17. R. Resco, L. W. Small and J. T. Stafford, Krull and global dimensions of semiprime Noetherian PI-rings, Trans. Amer. Math. Soc. 274 (1982), 285-295.

18. J. T. Stafford, On the ideals of a Noetherian ring, preprint.

Department of Mathematics, University of WAShington, SEATtLe, WashingTON 98195

Department of Mathematics, University of Glasgow, Glasgow, SCOtLAND 